Of course, $43^{\circ} \mathrm{N}$ is the latitude at which the boundary between the African and Eurasian plates meets the mid-Atlantic ridge, and so life there is not likely to be easy. Even so, the fact is that the supposed change in spreading rate 4 million years ago is observed in neither plate.

How, then, may this controversy be resolved? Kasameyer et al. (J. Geophys. Res., 77, 2535; 1972) have apparently succeeded by turning to experimental and theoretical studies of heat flow and bathymetry. The experimental data were obtained during a crossing of the ridge at $43^{\circ} \mathrm{N}$ by the research vessel Atlantis 2, the heat flow profile being the first ever taken across the mid-Atlantic ridge between $25^{\circ} \mathrm{N}$ and the Reykjanes ridge. In general, this profile is typical of oceanic ridge heat flow profiles. Thus the eight values of heat flow obtained at distances greater than $500 \mathrm{~km}$ from the central rift were uniform, all lying within 8 per cent of $1.45 \mu \mathrm{cal} \mathrm{cm} \mathrm{cm}^{-2} \mathrm{~s}^{-1}$ (h.f.u). This average is significantly higher than the 1.1 to 1.2 h.f.u. previously given for the North Atlantic (but with few values north of $40^{\circ} \mathrm{N}$ ), but is not out of line with that for the equatorial Atlantic. The eight values obtained within $120 \mathrm{~km}$ of the central rift were, however, higher on average (3.4 h.f.u.) and more variable (0.6 to 7.2 h.f.u.). On the other hand, the new profile differs from a typical profile in one important respect. At distances of 100 to $300 \mathrm{~km}$ from the crest it lacks symmetry, values in the west being some 60 to 80 per cent higher than in the east.

On the theoretical side, Kasameyer and his colleagues have adopted a model put forward some years ago by McKenzie ( $J$. Geophys. Res., 72, 6261; 1967) which makes possible the determination of heat flow and increased elevation caused by the creation of new lithosphere at the ridge crest. On the assumptions that the spreading rate is constant and that the temperature at the centre of the lithosphere is the same as that at the bottom, this model gives a steady-state heat flow equation which contains terms representing the heat carried by the moving slab and the heat transmitted by conduction. The temperature gradient at the surface is then used to calculate the extra heat flow (as a function of distance from the crest) due to the intrusion of hot magma into the lithosphere; a constant coefficient of thermal expansion is assumed in predicting the elevation.

Experience in the south Atlantic shows that this thermal model, although simple, predicts both bathymetry and gravity that agree well with reality; and so Kasameyer et al. feel justified in using it to solve the problem at $43^{\circ} \mathrm{N}$. Complications arise, however, in cases where the spreading rate is not constant. In such situations it is necessary to seek an approximate solution to the heat flow equation by assuming that all heat is carried away from the ridge by the moving slab-in other words, carried heat and vertical conduction are allowed, but horizontal conduction is considered negligible. As it turns out, this condition is not particularly restrictive. The simplified heat flow equation thus produced is highly insensitive to spreading rate (at least over the range 0.75 to $2.00 \mathrm{~cm} \mathrm{yr}^{-1}$ ), and has the advantage that the heat flow and elevation predicted from it depend only on the time since intrusion. In short, "the thermal history of a section of lithosphere depends on its age alone, not on the spreading history of the ridge. The thermal history is frozen into the lithosphere, much as the magnetic anomalies are frozen into the lithosphere." This means that Kasameyer and his colleagues have been able to plot predicted heat flow and bathy- metric profiles for $43^{\circ} \mathrm{N}$ based on the magnetic anomaly version of events, on the one hand, and based on the plate tectonic version, on the other. In other words, variations in spreading rate are accommodated easily by predicting heat flow and bathymetry at any given point solely from the age of the lithosphere there.

So what happens when predicted and observed profiles are compared? In practice, it turns out that the heat flow profiles are unsuitable for this comparison, partly because the predicted shape is not greatly affected by the 7 million year period of high spreading rate, partly because of difficulty in accurately determining the base line (even far from the ridge the excess heat flow due to lithospheric intrusion may not be negligible), and partly because the observed profile may be distorted by non-conductive heat transfer processes such as the circulation of water and

\title{
Detecting Human Anti-CEA Antibodies
}

Carcinoembryonic antigen (CEA) was first detected by Gold and Freedman in 1965 (J. Exp. Med., 121, 439) in adenocarcinomas of the human alimentary tract and in early foetal gut. It is a glycoprotein and a probable constituent of the tumour cell glycocalyx. The current clinical interest in CEA for the immunodiagnosis of cancer depends on the fact that this substance can be detected by a variety of radioimmunoassays in the peripheral blood of patients with cancer of the digestive system. When first describing it, Gold and Freedman referred to CEA as a tumour-specific antigen. By analogy with experimental animal tumours one would therefore expect CEA to evoke the appropriate specific responses in the host. The evidence on this point has so far been contradictory. In 1967 Gold (Cancer, 20, 1163) claimed that 70 per cent of patients with gut tumours and 70 per cent of pregnant women had in their serum an IgM anti-CEA antibody. These results were obtained with a somewhat unsophisticated haemagglutination technique. Burtin and his group (Immunology, 10, 507 ; 1966) showed, however, that such antibodies could be removed from the serum by absorption with normal gut extracts.

In next week's Nature New Biology (September 13) Gold, Freedman and Gold report a series of experiments using a very sensitive method for detecting antibodies, based on radiolabelled CEA. Their method involved mixing ${ }^{125} \mathrm{I}$ labelled CEA with the test serum for 48 $\mathrm{h}$, and then subjecting this mixture to immunoelectrophoresis with subsequent autoradiography. They examined 92 sera obtained from patients and normal healthy individuals. The presence of antibodies reacting with the labelled CEA was readily confirmed in both cancer patients and in normal subjects. The presence of these antibodies, predominantly IgM, appeared to correlate with $\mathrm{ABO}$ blood group status. Of 56 sera from individuals of blood group $\mathrm{O}$ or $\mathrm{B}, 80$ per cent contained detectable antibody. Of the remaining 36 sera from subjects of groups $A$ and $A B$, only 19 per cent contained anti-CEA activity. The sera were then absorbed with group A erythrocytes and the assay repeated. In the group $\mathrm{O}$ and $\mathrm{B}$ sera the anti-CEA activity was removed by absorption in all but two. In the $A$ and $A B$ population the activity was not affected. Purified human anti-A antibody was shown to react with CEA. Monospecific goat anti-CEA antiserum did not, however, react with blood group A substance. The sera from which anti-CEA activity was not absorbed out with group A red cells were all from patients with disseminated cancer.

These findings indicate an extensive cross-reaction between blood group A substance and their preparation of CEA. Antibody titration experiments suggest that this is a genuine cross-reaction, and was not due to contamination of the CEA preparation. But as the principal immunoreactant in A substance, $\mathrm{N}$ acetyl D-galactosamine, is absent from CEA, simple explanations for the crossreactivity will not suffice. The authors even postulate that the group A reactivity may be an artefact.

The presence of anti-CEA antibodies, not absorbed by group A red cells, in the sera of some cancer patients lends weight to the idea that CEA is a tumourspecific antigen and that it may even play a part in host resistance. 